\title{
BOS-Based Three-Dimensional Reconstruction of Rotor Blade Tip Vortex Positions
}

\author{
Nicola Debernardis ${ }^{(凶)}$, Clemens Schwarz, and Johannes N. Braukmann \\ German Aerospace Center (DLR), Institute of Aerodynamics and Flow Technology, \\ Bunsenstraße 10, 37073 Göttingen, Germany
}

\begin{abstract}
The present work discusses a methodology to reconstruct three-dimensional positions of rotor blade tip vortices based on stereoscopic background-oriented schlieren data. The reconstruction procedure is first applied on a rotor test stand where a comparison with previous PIV measurements is performed to validate the reconstruction scheme. Subsequently, it is adapted for the reconstruction of the vortex system surrounding a model helicopter in free flight during an unsteady takeoff maneuver.
\end{abstract}

Keywords: Epipolar geometry $\cdot 3 \mathrm{D}$ reconstruction $\cdot$ BOS $\cdot$ Blade tip vortices

\section{Introduction}

Over the last years many investigations on rotor blade tip vortices have been carried out in the Institute of Aerodynamics and Flow Technology in Göttingen using optical measurement techniques. In two recent experiments particle image velocimetry (PIV) and the background-oriented schlieren technique (BOS) were combined to investigate the wakes of an isolated rotor on a test stand under cyclic pitch conditions [1] and of a free-flying model helicopter in ground effect [2]. In the present investigation, the BOS data acquired in both experiments are used to reconstruct the corresponding vortex filaments in three-dimensional (3D) space. In addition to its visualization intent, BOS is tested as a method for the extraction of quantitative values. Due to its easier applicability, it constitutes an efficient alternative for the determination of three-dimensional vortex positions in comparison to a volumetric PIV setup. In both cases, the vortex systems were recorded simultaneously from different perspectives using stereo camera systems. By using epipolar geometry it is possible to match discrete vortex points and subsequently triangulate their position in 3D space. This approach has been used in several studies in the past to reconstruct tip vortices of both fixed wing aircraft [4] and helicopters in wind tunnel measurements [5] and in free flight $[3,6]$. In the current study a modular reconstruction scheme was developed that allowed for the analysis of datasets recorded with different experimental setups

(C) The Author(s), under exclusive license to Springer Nature Switzerland AG 2021

A. Dillmann et al. (Eds.): STAB/DGLR Symposium 2020, NNFM 151, pp. 315-324, 2021.

https://doi.org/10.1007/978-3-030-79561-0_30 
for a multitude of test cases. The methodology was applied to data from the rotor test stand and the model helicopter to analyze the spatial structure of rotor blade tip vortices in different conditions.

\section{Rotor Test Stand Experiment (RTG)}

\subsection{Experimental Setup at the RTG}

The experiment was carried out at the rotor test facility in Göttingen (RTG) [1]. The four-bladed rotor had a radius of $R=0.65 \mathrm{~m}$, a chord length of $c=0.072 \mathrm{~m}$, a DSA-9A airfoil and was arranged horizontally (see Fig. 1), (see [1] for details). The rotor was operated with collective and cyclic pitch settings (reported in [1]) in a wind tunnel which provided a slow axial inflow of $2.5 \mathrm{~m} / \mathrm{s}$. The combination of cyclic pitch and an axial inflow represents a generic test case. The cyclic pitch enables to study the resulting aerodynamics in a highly unstable rotor downwash, while the axial inflow ensures known inflow conditions as explained in [1]. With this setup the rotor was operated out of ground effect. A detailed description of the RTG can be found in [7]. The measurement techniques used during the experiment are PIV, to characterize the velocity field, and BOS to acquire volumetric information around the PIV plane.

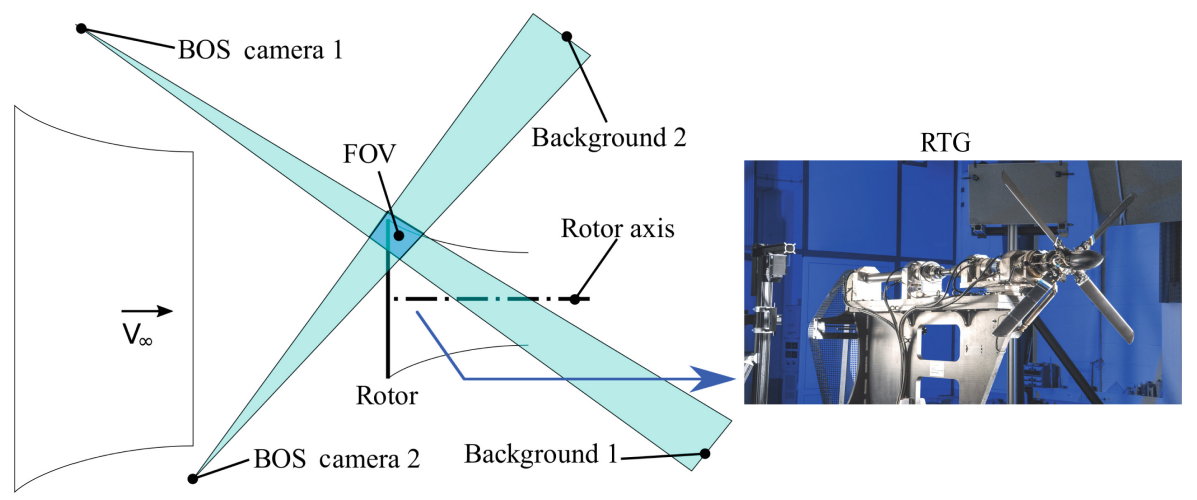

Fig. 1. Sketch of the RTG (top view) with the BOS setup (left) and image of the RTG (right, after [1]). In addition, a sketch of the field of view (FOV) is included.

The BOS cameras were imaging a measurement volume around the PIV plane covering an azimuthal range of $22^{\circ}$ (see Fig. 1). A high focal length of $f=300$ $\mathrm{mm}$ was chosen to increase the sensitivity of the setup to density gradients.

\subsection{Reconstruction Methodology}

Figure 2 depicts sample BOS results recorded instantaneously with both cameras. The BOS result after the correlation is a displacement field of the background pattern. The vortices are visualized by means of the gradient of the displacement field. 
The individual vortex filaments appear as bright lines. To detect the vortex filaments in the images, the NeuronJ neurite tracing algorithm designed by Meijering [8] has been used which was applied for the extraction of vortices in [1,12]. The implementation of this tool to the tracing of blade tip vortices was first shown by Overmeyer [10], who successfully traced vortices in shift images of different signalto-noise ratios (SNR). The points on the vortex filaments, identified with NeuronJ in the image of camera 1 (yellow line), generate a pencil of epipolar lines in the second image. The epipolar lines can be used to establish corresponding points on a vortex filament of camera 1 in the corresponding image of camera 2 . This is done by intersecting the epipolar lines from the first camera (orange line in Fig. 2 b) with the vortex filaments in the second camera.

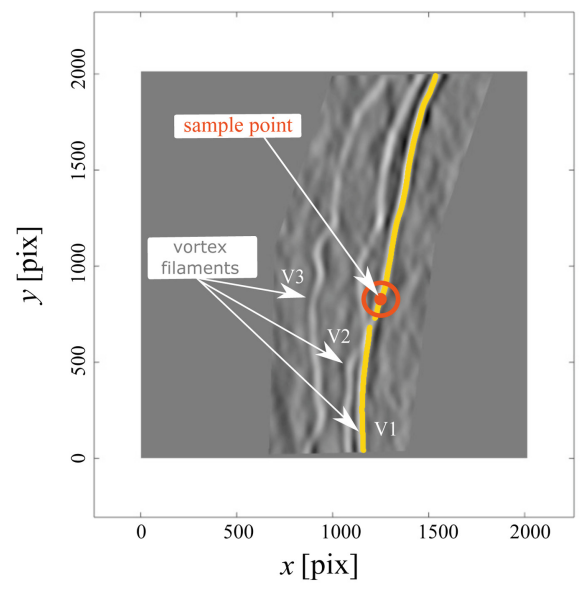

(a)

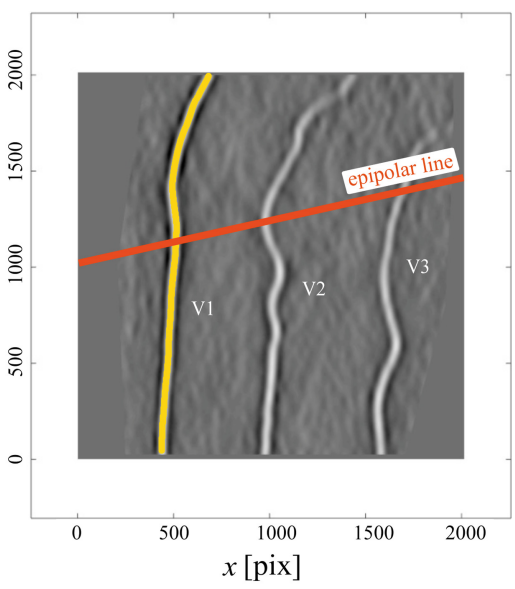

(b)

Fig. 2. Sample BOS result from camera 1 (a) and 2 (b) with epipolar line for a point on vortex filament V1 in the corresponding image from camera 2. During the acquisition the blade loading was $\frac{C_{T}}{\sigma}=0.157$ and the angular velocity was set to $\Omega=2 \pi \cdot 23.6 \mathrm{~Hz}$

A fundamental requirement for both the application of epipolar geometry and for the triangulation of points in space is a reliable calibration of the camera system. The calibration was performed using the camera calibration toolbox for MATLAB by Bouguet [11]. A calibration target consisting of a dot pattern was used, the detected markers were used as input for the calibration. The calculated camera matrices serve to derive projection matrices $P$ and $P^{\prime}$ and subsequently construct the fundamental matrix for the stereo setup. The accuracy in establishing point correspondence was estimated referring to the position of well known targets and resulted in 1.14 pixel $( \pm 0.26 \mathrm{~mm})$. 


\section{$2.3 \quad$ Results}

The 3D locations of the vortices were triangulated for a number of four test cases. Every selected test case corresponds to a different pitch phase $(t / T)$ at a different azimuthal position, but the same inflow conditions. During each revolution the blades undergo one full pitch cycle. The pitching variation was $\Theta=24^{\circ}-6^{\circ} \cos (2 \pi t / T)$. For each test case, a total of 200 images for each camera were taken. A total of four test cases has been analyzed covering wake ages ranging from $\Psi_{v}=40^{\circ}$ to $\Psi_{v}=70^{\circ}$. The first two correspond to wake ages of $40^{\circ}$ and $55^{\circ}$ relative to blade 1 . The two other test cases, on the other hand, contain identical wake ages with respect to blade 3 . Since the two sets correspond to different phases during the pitch cycle, the corresponding vortices were created at different pitch angles. The same four cases were measured simultaneously with PIV allowing for a direct comparison between the vortex positions. Figure 3a depicts the resulting vortex locations for a sample data set. The lighter lines represent the individual filaments, whereas the darker lines correspond to the phase average of all the 200 vortex filaments. The averaged vortex locations for all four analyzed test cases are shown in Fig. $3 \mathrm{~b}$ in a perspective view. The three vortices in the upper right corner were created during the down-stroke motion of the blade, where the blade goes from a pitch phase of $t / T=0.88$ to $t / T=0.96$. The other two vortices on the opposite side were created during an up-stroke motion where the blade goes from a pitch phase of $t / T=0.38$ to $t / T=0.47$.

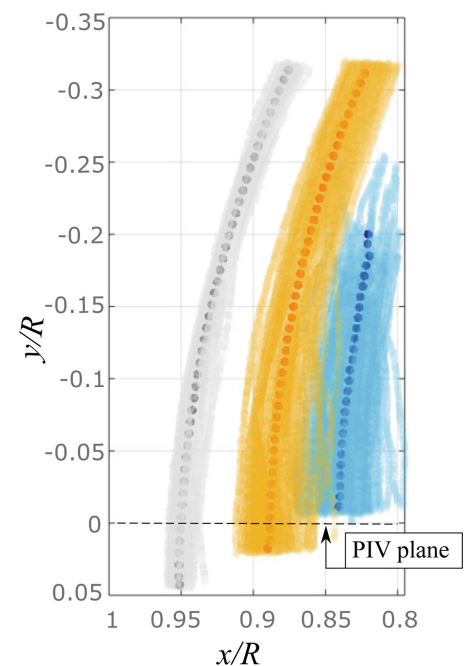

(a)

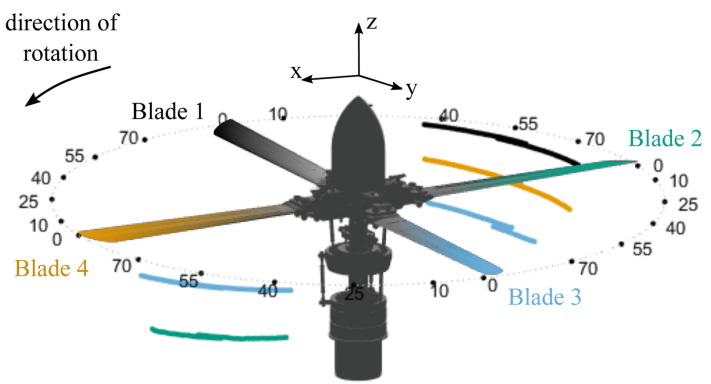

(b)

Fig. 3. Reconstructed vortex positions from 200 images for a sample test at $t / T=0.96$ and $\Psi_{v}=40^{\circ}(3 \mathrm{a})$ and $3 \mathrm{D}$ representation of all four analyzed test cases $(3 \mathrm{~b})$. Each vortex is colored based on the blade it originated from. 
The reconstructed blade tip positions allow for a determination of the rotor hub position and a new local coordinate system $(x, y, z)$ is defined by moving the origin into the rotor hub center. The vortices are color coded based on the blade they trail from. The vortices with a higher wake age have a greater vertical convection rate that induces larger distances between the vortex filaments.

\subsection{PIV Comparison}

To validate the reconstruction process, the vortex positions extracted from BOS data are compared to simultaneously recorded PIV measurements. The PIV measurements were taken at the same relative wake ages of $40^{\circ}$ and $55^{\circ}$ and the same phase $(t / T)$.

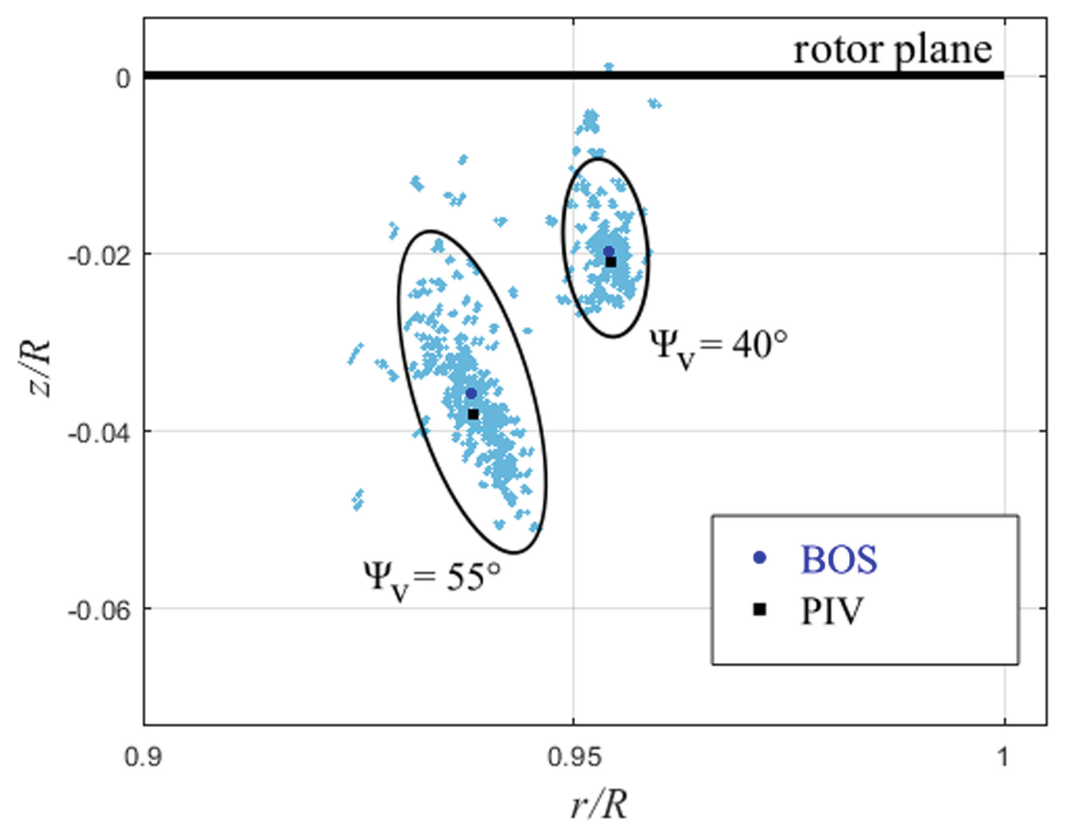

Fig. 4. Instantaneous and mean vortex positions in a radial plane (r, z). The vortex positions refer to phases of $t / T=0.96$ and $t / T=0.92$ and wake ages of 40 and $55^{\circ}$ respectively.

Figure 4 depicts individual and average vortex positions from BOS (blue) and the average vortex center that was determined based on velocity data from PIV (black) [1]. The vortex positions refer to phases of $t / T=0.96$ and $t / T=0.92$ at two different wake ages of $40^{\circ}$ and $55^{\circ}$ relative to blade 1 . In addition, ellipses representing a confidence interval of $95 \%$ are included. It can be seen that as the wake age increases the radial vortex position decreases. The cycle-to-cycle scatter in individual vortex positions is inherent to a rotor tip vortex system and was analogously observed in the PIV data [1]. The average locations show a deviation 
of only $0.09 \mathrm{~mm}$ between BOS and PIV. This suggests that stereoscopic BOS can be a valuable tool to gain quantitative information on the spatial structure of a vortex system.

\section{Free-Flying Model Helicopter Experiment}

\subsection{Experimental Setup of the Model Helicopter}

In the second experiment, the wake of a free-flying model helicopter in ground effect was investigated. A series of flight tests were performed consisting of both quasi-steady and unsteady maneuvering flight states. A more detailed discussion of the different flight states and the resulting wake structure is given in [2]. The tests were conducted with the DAEDALUS model helicopter from the Institute of Aerodynamics and Gas Dynamics at the University of Stuttgart, shown in Fig. 5. The test rotorcraft is a model helicopter with two untwisted and stiff rotor blades with a radius of $R=0.7 \mathrm{~m}$, chord length of $c=54.5 \mathrm{~mm}$, and a symmetrical Joukovski 0015 airfoil. In addition to the PIV measurements reported in [2], a multi-camera stereo-BOS system was used to capture the spatial structure of the vortex system surrounding the helicopter. A sketch of the setup is shown in Fig. 5 along with a photograph of the test helicopter and sample BOS results from both perspectives for a takeoff maneuver. Further details on the BOS setup can be found in [9].

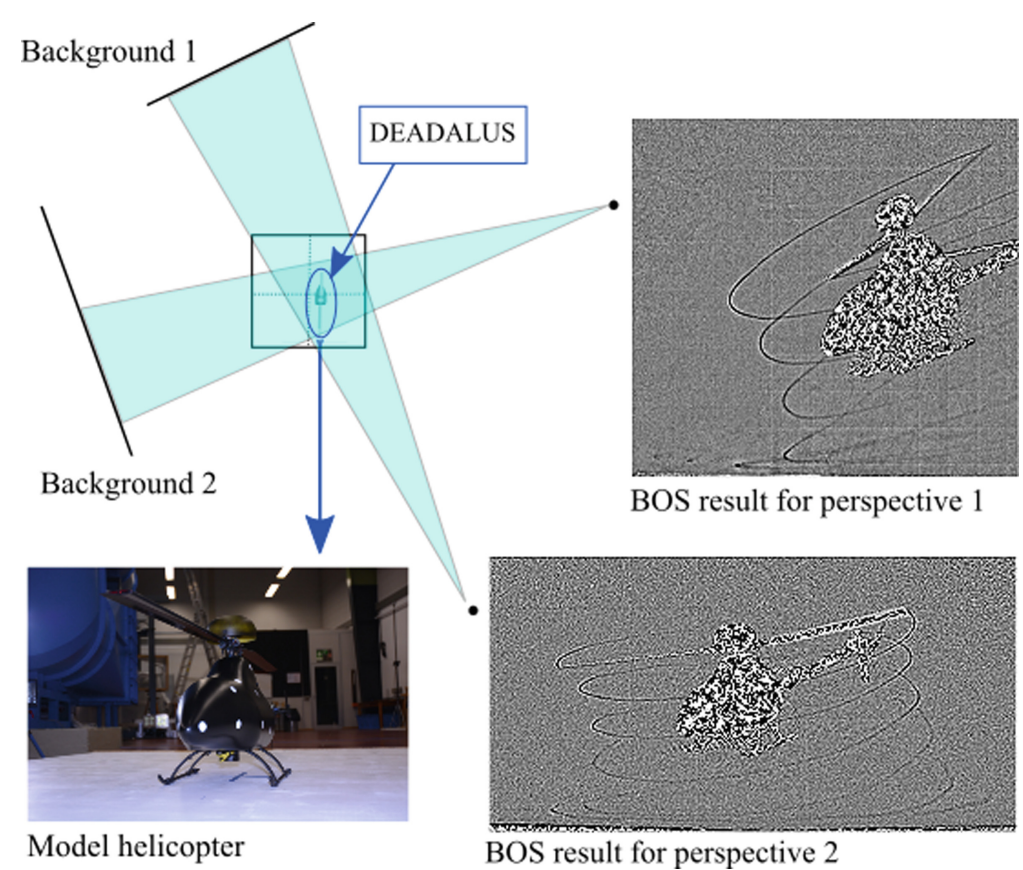

Fig. 5. Top view of the experimental setup along with a photograph of the test helicopter and sample BOS results for both camera perspectives during a takeoff maneuver. 


\subsection{Reconstruction Methodology}

Figure 6 depicts the vortex system for both camera perspectives during a takeoff maneuver. Analogous to the RTG experiment, a reconstruction scheme based on epipolar geometry was applied. A difficulty in identifying corresponding vortex points arises when the vortex axis is nearly parallel to the epipolar line. The green epipolar line in Fig. 6b, for example, intersects the second vortex in two points. In the case of multiple intersection points, false pairing of points on non-corresponding vortices have to be checked manually for their physical validity. While in the case of two discrete and identifiable intersection points this problem can still be solved, the more the epipolar line aligns to the vortex axis, the more the limit in the accuracy is approached. Thus, the reconstruction of adjacent points is limited by the possibility of identifying a univocal intersection point. Another limiting factor is the low SNR in certain regions. This limits especially the reconstruction of older vortices due to their decreasing strength. In the present case only vortices up to a wake age of about $650^{\circ}$ could be reconstructed.

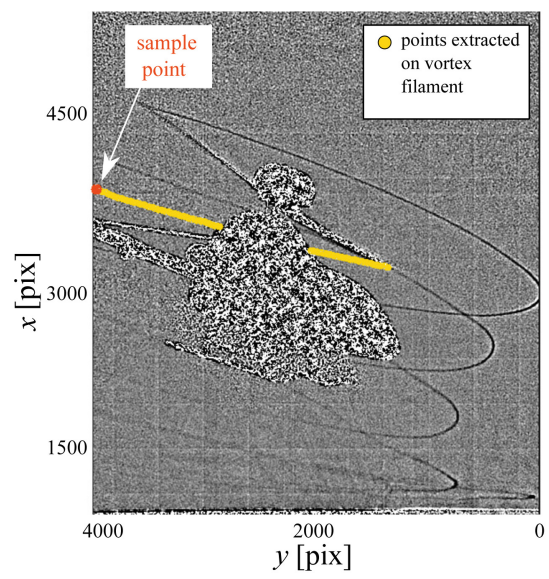

(a)

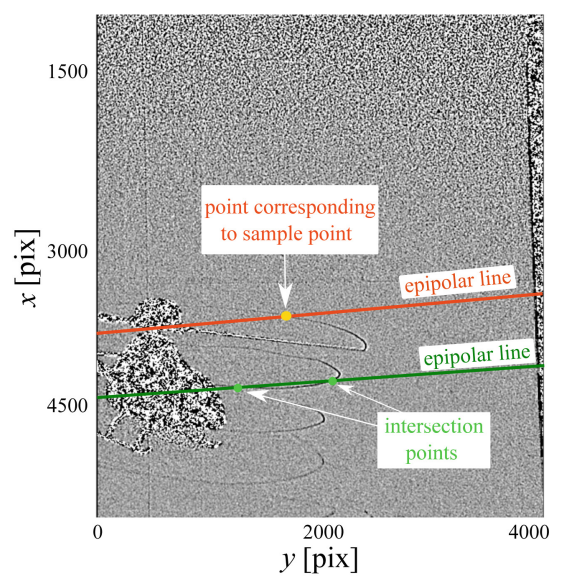

(b)

Fig. 6. BOS results from both camera perspectives showing the epipolar intersection with a vortex filament. The individual orange dot on the yellow vortex filament (image 6a), corresponds to the orange epipolar line (6b). The yellow intersection point with the vortex filament corresponds to the orange sample point in image $6 \mathrm{a}$.

\subsection{Results}

Despite the increased complexity, it was possible to reconstruct significant parts of the vortex system, which are depicted in Fig. 7. For visualization purposes, the whole vortex system is depicted together with a 3D model of the DAEDALUS model helicopter in multiple views. A new local reference system $(x, y, z)$ is 
defined by moving the origin to the rotor hub center. The vortices are color coded based on the blade they trail from. The model helicopter is added to the plots with its position and orientation determined by triangulating optical markers that were applied to the fuselage (see [2] and [9]). The rotor blade orientation is determined by the reconstructed locations of the blade tips, assuming a stiff rotor. While for the first blade on the advancing side, the blade tips vortex filament match the blade tip where it trails from, for the retreating blade an offset between blade tip and vortex can be detected. This vertical offset can be due to the bending of the blade, which is not accounted for in the 3D model.

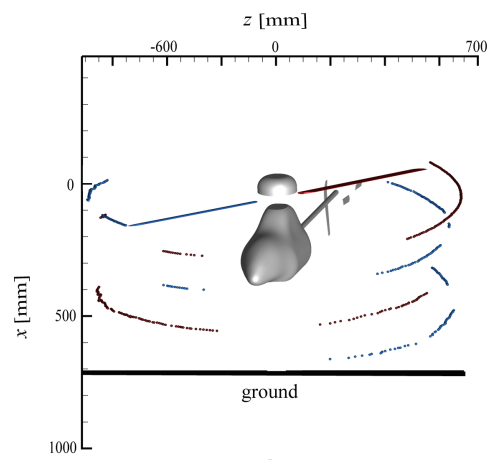

a

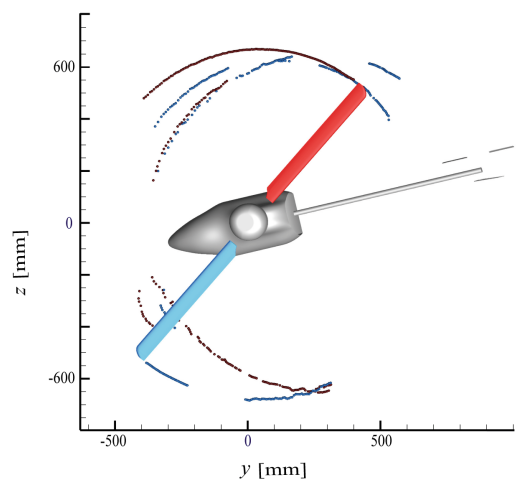

$\mathrm{c}$

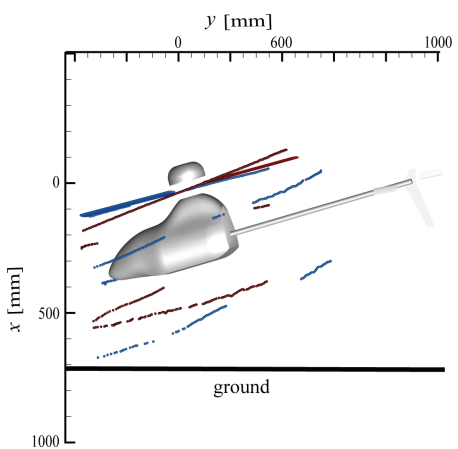

b

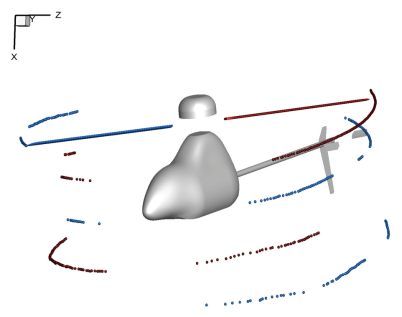

d

Fig. 7. Different perspectives $(\mathrm{a}-\mathrm{d})$ of the model helicopter and reconstructed main rotor vortex system.

The characteristic helical shape of the vortex system is visible. After a first contraction of the wake, a subsequent radial expansion with wake age due to the ground effect occurs. At the time of the image acquisition, the helicopter rotor is about 1 rotor radius $R$ above the ground as was reported in [2]. The evolution of the vortex system's radius versus the wake age is better visible in Fig. 8. After a first contraction, which reaches a value of $0.9 R$ at the wake age of $340^{\circ}$, the 
radius of the vortex system expands due to the proximity of the ground starting from a wake age of $470^{\circ}$ up to the maximum detectable wake age of $650^{\circ}$. A similar behavior was also observed in data from PIV measurements for the same model helicopter in ground effect [2].

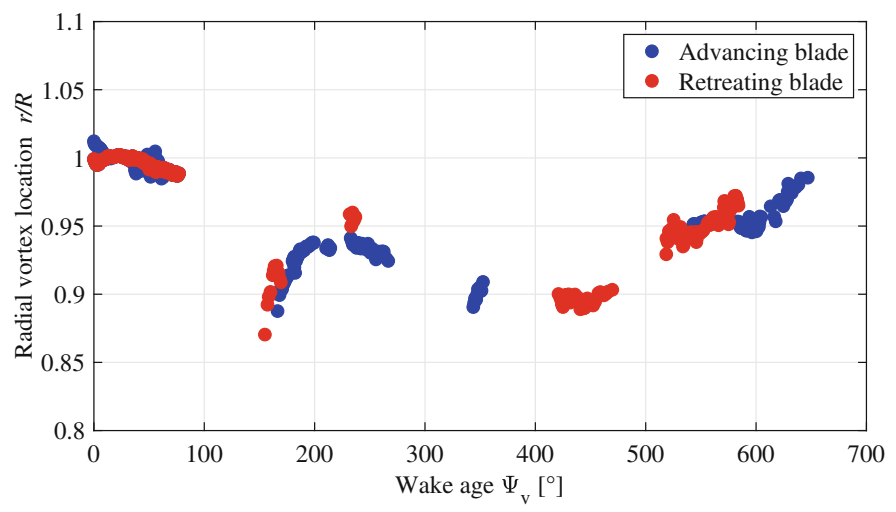

Fig. 8. Radius of the vortex system over wake age.

\section{Conclusion}

A procedure for 3D vortex reconstruction based on stereoscopic BOS data was presented and applied to two different experiments. In the first part of the study, the vortex system produced by a rotor test stand with cyclic pitch was investigated. Based on a calibrated stereo camera system, fundamental matrices were determined that allowed for the use of epipolar geometry in order to establish point correspondences between the images of two cameras. Individual points on the vortex filaments were matched by calculating epipolar lines for vortex points in the first image and extracting the intersection point with the corresponding vortex filament in the second image. The accuracy of the epipolar based reconstruction was quantified by reconstructing the 3D position of a target whose geometry in 3D space was known with good precision. The comparison with PIV mean vortex positions (see [1]) allowed the validation of the reconstruction process that was further applied to a second experiment. In the second part of the current investigation, the reconstruction scheme was applied to the vortex system of a free-flying model helicopter in ground effect. Despite the low SNR ratio for older vortices and the unfavorable geometric setup with regard to vortex point identification based on epipolar geometry, it was possible to reconstruct significant parts of the vortex system up to a wake age of $650^{\circ}$. Overall, the results showed the feasibility and the value of a $3 \mathrm{D}$ reconstruction of vortex filaments from stereo-BOS data. The developed reconstruction scheme allows for a quantitative analysis of large parts of the rotor vortex system and can be applied to an extended database in the future. 


\section{References}

1. Braukmann, J.N., Wolf, C.C., Goerttler, A., Raffel, M.: Blade tip vortex system of a rotor with cyclic pitch. AIAA J. 58(2020). https://doi.org/10.2514/1.J058678

2. Schwarz, C., Bauknecht, A., Mailänder, S., Raffel, M.: Wake characterization of a free-flying model helicopter in ground effect. J. Am. Helicopter Soc. 64(012010) (2019) https://doi.org/10.4050/JAHS.64.012010

3. Klinge, F., Hecklau, M., Raffel, M., Kompenhans, J., Göhmann, U.: Measurement of the position of rotor blade vortices generated by a helicopter in free flight by means of stereoscopic Background Oriented Schlieren Method (BOS). In: 13th International Symposium on Application of Laser Techniques to Fluid Mechanics, Lisbon, Portugal, 26-29 June (2006)

4. Meyn, L.A., Bennett, M.S.: Application of a two camera video imaging system to three-dimensional vortex tracking in the 80 -by 120 -foot wind tunnel. In: AIAA 11th Applied Aerodynamics Conference, Monterey, CA, USA, 1 January (1993)

5. Schairer, E., Kushner, L.K., Heineck, J.T.: Measurements of tip vortices from a full-scale UH-60A rotor by retro-reflective background oriented Schlieren and stereo photogrammetry. In: American Helicopter Society 69th Annual Forum, Phoenix, AZ, USA, 21-23 May 2013

6. Bauknecht, A., Ewers, B., Wolf, C.C., Leopold, F., Yin, J., Raffel, M.: Threedimensional reconstruction of helicopter blade-tip vortices using a multi-camera BOS system. Exp. Fluids 56(1), 1-13 (2014). https://doi.org/10.1007/s00348-0141866-6

7. Schwermer, T., Richter, K., Raffel, M.: Development of a rotor test facility for the investigation of dynamic stall. In: New Results in Numerical and Experimental Fluid Mechanics X, vol. 132. Springer, Cham (2016) https://doi.org/10.1007/9783-319-27279-5_58

8. Meijering, E., Jacob, M., Sarria, J.-C.F., Steiner, P.L., Hirling, H., Unser, M.: Design and validation of a tool for neurite tracing and analysis in fluorescence microscopy images. Cytom. Part A J. Int. Soc. Anal. Cytol. 58 (2004). https:// doi.org/10.1002/cyto.a.20022

9. Bauknecht, A., Schwarz, C., Raffel, M., Mailänder, S.: Flow measurement techniques for rotor wake characterization on free-flying helicopters in ground effect. In: AIAA Scitech Forum, San Diego, CA, USA (2019). https://doi.org/10.2514/6. 2019-2107

10. Overmeyer, A.D.: A rotor tip vortex tracing algorithm for image post-processing. In: American Helicopter Society 71st Annual Forum, Virginia Beach, VA, USA, May 5-7 (2015)

11. Bouguet, J.Y.: Camera Calibration Toolbox for Matlab. http://www.vision. caltech.edu/bouguetj/calib_doc/index.html. Accessed 32020

12. Schwarz, C., Bauknecht, A., Wolf, C.C., Coyle, A., Raffel, M.: A full-scale rotorwake investigation of a free-flying helicopter in ground effect using BOS and PIV. J. Am. Helicopter Soc. 65(032007) (2020). https://doi.org/10.4050/JAHS.65.032007 